\title{
Polarization Mode Dispersion in Birefringent Optical Fibers
}

\author{
T.R. WOLIŃSKI* AND A.W. DOMAŃSKI \\ Faculty of Physics, Warsaw University of Technology \\ Koszykowa 75, 00-662 Warsaw, Poland
}

\begin{abstract}
The paper discusses influence of polarization mode dispersion on performance of polarimetric systems with highly birefringent fibers. It appeared that polarization mode dispersion strongly influences a degree of polarization that depends on coherence of the light source used and simultaneously diminishes dynamics of the output signal.
\end{abstract}

PACS numbers: 42.81.Gs, 42.81.Pa, 42.25.Ja, 42.25.Dd

\section{Introduction}

Polarization effects in optical fibers were recognized many years ago and have been used in developing polarimetric sensors utilizing highly birefringent (HB) polarization-maintaining (PM) fibers [1]. However, in telecommunication they have initially played a minor role in the development of lightwave systems as long as single channel bit-rates were below 2.5 Gbit/s. Due to enormous increase in optical path lengths and also increase in bit rates in digital systems beyond $10 \mathrm{Gbit} / \mathrm{s}$, it appeared that a new obstacle, known as polarization mode dispersion (PMD), is regarded as a major limitation in optical transmission systems in general and an ultimate limitation for ultra-high speed single channel systems based on standard single mode fibers.

The degree of polarization (DOP) of the light propagating in an HB optical fiber diminishes along the propagating distance. This effect is of particular interest since commonly used quasi-monochromatic semiconductor laser sources are not perfectly coherent. It has been shown [2,3] that the DOP measurement can be used to determine the coherence characteristics of laser diodes. This issue is of great interest not only in telecommunication applications but also in polarimetric sensors with HB fibers.

* corresponding author; e-mail: wolinski@if.pw.edu.pl 
The paper discusses the importance of PMD in HB fibers in comparison to single-mode telecom fibers and also addresses the problem of DOP fading in polarimetric fiber optic systems with HB fibers. Some recent experimental results of the measurements of different polarization characteristics for HB fibers with intrinsic but also with dynamically controlled birefringence are also presented.

\section{Origin of polarization mode dispersion in optical fibers}

Polarization mode dispersion originates from the internal birefringence due to either asymmetry of the fiber optic cross section or anisotropic stresses acting on the core of the fiber. Asymmetry is caused by the fact that the fiber core is slightly not round or elliptical. When the core of the fiber is asymmetrical, the light polarized along a birefringence axis moves slower than the light polarized along the orthogonal axis. In real single-mode fibers, which possess nonzero internal birefringence, both orthogonally polarized modes have randomly different phase velocities, causing fluctuations of the polarization state of the light guided in the fiber. This effect can spread the pulse enough to make it overlap with other pulses or change its own shape enough to make it undetectable at the receiver.

Since optical fibers allow very large propagation distances even very small birefringence effects can cumulate along fiber and their random distribution over the large lengths causes polarization properties of guided light generally difficult to determine. It concerns both the state and the degree of polarization, and consequently PMD is a stochastic process.

Polarization mode dispersion, usually expressed by differential group delay (DGD) over the length of the fiber $\Delta \tau / L$ and modal birefringence $\Delta \beta$ are the most important parameters characterizing birefringent fibers. Both parameters are interrelated according to the following formula:

$$
\frac{\Delta \tau}{L}=\frac{\mathrm{d}(\dot{\mathrm{C}} \beta)}{\mathrm{d} \omega}=\frac{1}{c}\left(\Delta n_{\mathrm{eff}}+\omega \frac{\mathrm{d} \Delta n_{\mathrm{eff}}}{\mathrm{d} \omega}\right),
$$

where $\Delta \tau / L$ is usually expressed in units of picoseconds per kilometer of fiber length, $\Delta n_{\mathrm{eff}}$ is the differential effective index of refraction for the slow and fast polarization modes, and $\omega=2 \pi c / \lambda$ is the angular frequency of light.

For the HB bow-tie fibers in which birefringence is caused by stress applying parts introduced in cladding close to the core region of the fiber, $\Delta n_{\text {eff }}$ is nearly wavelength independent and the chromatic dispersion of the modal birefringence is negligible. Hence for this type of fibers measurements of birefringence and PMD are equivalent

$$
\frac{\Delta \tau}{L} \cong \frac{1}{c} \Delta n_{\mathrm{eff}}=\frac{\lambda}{L_{\mathrm{B}} c},
$$

where $L_{\mathrm{B}}$ is beat length expressed as

$$
L_{\mathrm{B}}=2 \pi /\left|\beta_{y}-\beta_{x}\right|
$$


and responsible for phase difference changes along the HB fiber. The spatial period $L_{\mathrm{B}}$ of these changes reflects the modulation in the polarization states along the fiber [1]. Linearly polarized light coupled into the HB fiber with plane of polarization directed at the angle of 45 degrees between both axes of birefringence becomes elliptical polarized and next circular, elliptical, linearly orthogonal, elliptical, circular, elliptical and finally the same linearly polarized and oriented as introduced into fiber. Typical values of PMD for HB fibers are close to $1000 \mathrm{ps} / \mathrm{km}$, that is four orders of magnitude higher in comparison to $0.1 \mathrm{ps} / \mathrm{km}$ (typical values of PMD for telecommunication fibers).

In optical fiber systems influence of PMD on the system performance can be estimated by detecting changes in degree of polarization (DOP) of the light passing through the fiber. In general, PMD diminishes DOP of light that depends on coherence of the light source. Figure 1 presents schematically influence of PMD in both: telecom and HB fibers.

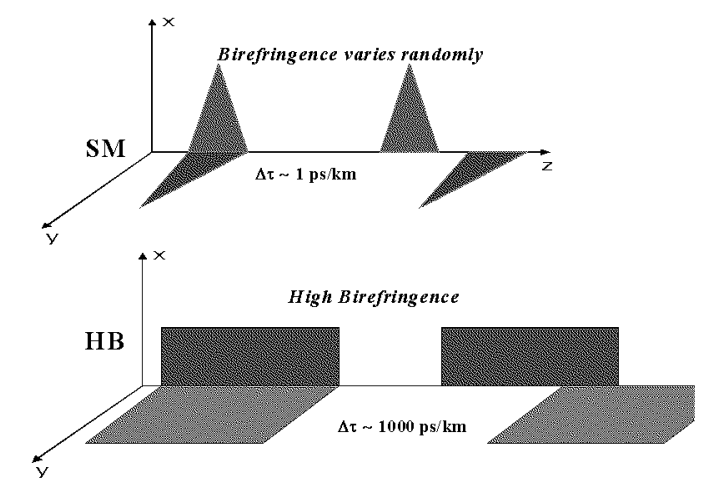

Fig. 1. Schematic representation of DOP degradation by PMD; upper: single-mode (SM) telecom fibers with low PMD, lower: HB fibers with high PMD.

According to Born and Wolf [4] the light in which the wavelength range $\Delta \lambda$ is small compared to the mean wavelength

$$
\Delta \lambda / \lambda \ll 1 \quad \text { or } \quad \Delta \omega / \omega \ll 1
$$

is called the quasi monochromatic light. To describe and analyze polarization phenomena in such a case the Stokes vector and the Mueller matrix formalism are necessary.

In the Mueller-Stokes formalism, the Stokes parameters have a simple physical interpretation and are related to intensity measurements. It is thus useful to gather these parameters in a four-component array, the Stokes vector $(\boldsymbol{S})$ : 


$$
S=\left[\begin{array}{c}
S_{0} \\
S_{1} \\
S_{2} \\
S_{3}
\end{array}\right]=\left[\begin{array}{c}
I_{x x}+I_{y y} \\
I_{x x}-I_{y y} \\
I_{x y}+I_{y x} \\
\mathrm{i}\left(I_{y x}-I_{x y}\right)
\end{array}\right],
$$

where $S_{0}, S_{1}, S_{2}, S_{3}$ are the Stokes vector parameters, $I_{0}\left(S_{0}\right)=I_{\mathrm{P}}+I_{\mathrm{u}}$ ( $I_{\mathrm{P}}$ signifies polarized, and $I_{\mathrm{u}}$ unpolarized light, respectively) is the total light intensity, $I_{x x}=\left\langle E_{x} E_{x}^{*}\right\rangle, I_{x y}=\left\langle E_{x} E_{y}^{*}\right\rangle, I_{y y}=\left\langle E_{y} E_{y}^{*}\right\rangle, I_{y x}=\left\langle E_{y} E_{x}^{*}\right\rangle$, and $\boldsymbol{E}$ is the electric field vector.

The $D O P$ can be straightforwardly defined by the Stokes parameters as:

$$
D O P=\frac{I_{\mathrm{P}}}{I_{0}}=\frac{\sqrt{S_{1}^{2}+S_{2}^{2}+S_{3}^{2}}}{S_{0}}
$$

\section{Polarization mode dispersion and nonlinearity}

Apart from linear (intrinsic or induced) birefringence optical fibers are also characterized by nonlinear effects associated with nonlinear birefringence that can also influence polarization mode dispersion $[1,5]$.

Nonlinear or self-induced birefringence relies on the nonlinear coupling between the orthogonally polarized components of an optical wave that changes the refractive index by different amounts of $\Delta n_{x}$ and $\Delta n_{y}$ due to nonlinear contributions

$$
\begin{aligned}
& \Delta n_{x}=n_{2}\left[\left|E_{x}\right|^{2}+\frac{2}{3}\left|E_{y}\right|^{2}\right], \\
& \Delta n_{y}=n_{2}\left[\left|E_{y}\right|^{2}+\frac{2}{3}\left|E_{x}\right|^{2}\right],
\end{aligned}
$$

where $n_{2}$ is the nonlinear-index coefficient given by the formula

$$
n_{2}=\frac{3}{8 n} \operatorname{Re}\left(\chi_{\chi \chi \chi \chi}^{(3)}\right)
$$

and defined by one component of the 4-rank nonlinear susceptibility tensor component $\chi_{\chi \chi \chi \chi \chi}^{(3)}$. In the case of silica fibers, where the dominant nonlinear contribution is of electronic origin, the nonlinear-index coefficient has a value of $3.2 \times 10^{-22} \mathrm{~m}^{2} / \mathrm{W}$.

As the wave propagates along the fiber, it acquires an intensity-dependent nonlinear phase given by

$$
\begin{aligned}
& \phi_{x}^{\mathrm{nl}}=\frac{2 \pi}{\lambda} L \Delta n_{x}=\frac{2 \pi}{\lambda} L n_{2}\left[\left|E_{x}\right|^{2}+\frac{2}{3}\left|E_{y}\right|^{2}\right], \\
& \phi_{y}^{\mathrm{nl}}=\frac{2 \pi}{\lambda} L \Delta n_{y}=\frac{2 \pi}{\lambda} L n_{2}\left[\left|E_{y}\right|^{2}+\frac{2}{3}\left|E_{x}\right|^{2}\right] .
\end{aligned}
$$


The first term in the brackets is responsible for self-phase modulation (SPM), while the second term results from the phase modulation of one polarization (wave) by the co-propagating orthogonal polarization (wave) and is responsible for so-called cross-phase modulation (XPM). The XPM-induced nonlinear coupling between the field components $E_{x}$ and $E_{y}$ creates nonlinear birefringence that changes the state of polarization (SOP) if the input light is elliptically polarized. The nonlinear coupling between the two orthogonally polarized components of the optical wave is referred as nonlinear or self-induced birefringence and has many device applications.

By choosing an appropriate pulse power and shape, the effects of chromatic dispersion and SPM can be balanced and this is an optical soliton. Optical solitons are not only robust to chromatic dispersion but also to disturbing effect of PMD. The two orthogonal polarization states that are differentially delayed as an effect of PMD, induce a nonlinear phase shift by the cross-phase modulation that mutually shifts one another's frequencies in opposite directions. Through the chromatic dispersion, the two polarization states will have different speeds that counteract the PMD. This phenomenon is called soliton-trapping and works optimally in HB polarization-maintaining fibers [5].

\section{Experimental results}

We have constructed the polarization analyzing system PAS-1 described elsewhere [6] to measure the state and the degree of polarization of the output light. The semi-automated polarization analyzing system can measure four light intensities, for four different arrangements of the analyzer and the quarter wave plate and then automatically calculates four Stokes vector components along with intensity of the output light as well as its DOP parameter.

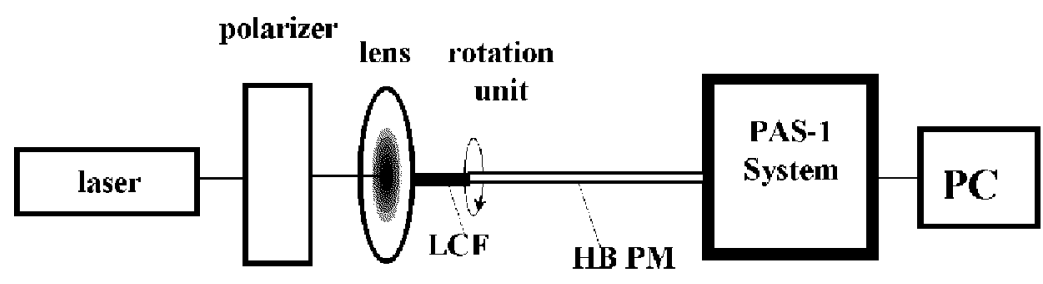

Fig. 2. Experimental setup to investigate polarization effects in the birefringent systems.

Figure 2 shows the experimental set-up for polarimetric measurements of the birefringence optical fibers. The linearly polarized light is launched into the bow-tie HB 600 fiber and then is analyzed by the PAS- 1 polarimeter. The polarimeter was also connected to a computer, which calculated the DOP parameter. Toshiba laser diode (TOLD-9321) operating at $670 \mathrm{~nm}$ wavelength was used as a light source 


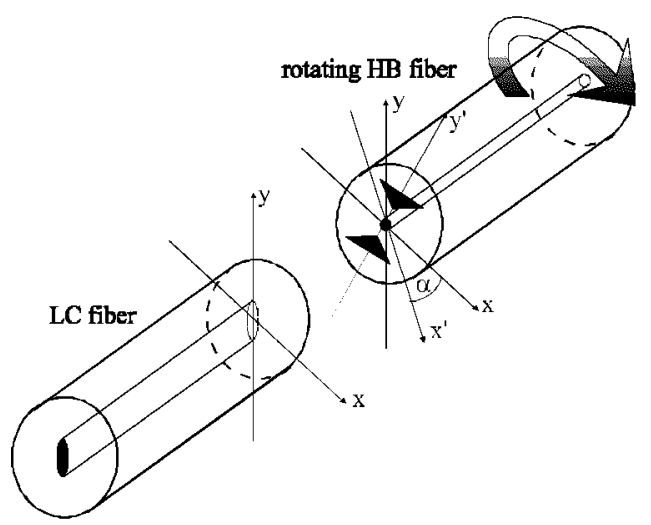

Fig. 3. Polarization phenomena in birefringent systems with different HB fibers.

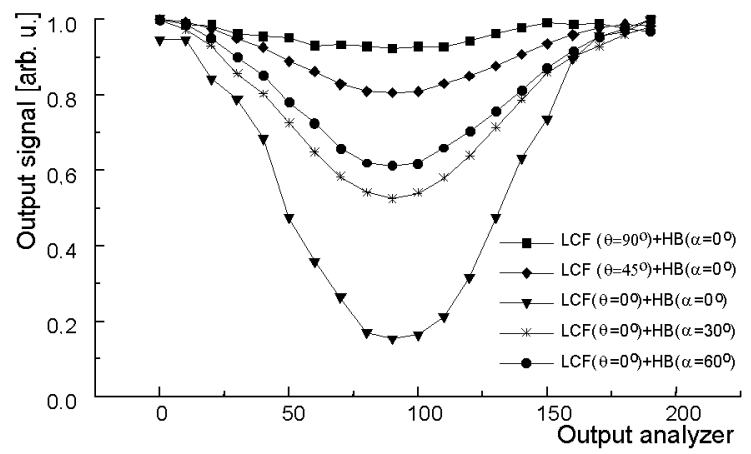

Fig. 4. Output signal as a function of the output polarization for different configurations of the LCF (1335-1; $5 \mathrm{~cm}$ long) and the rotated HB fiber (50 cm long), and for different directions of the input polarization.

and different types of HB fibers have been examined. These included standard silica bow-tie HB fibers but also liquid-crystal fibers (LCFs) with elliptical core in which birefringence changes can be dynamically induced and controlled. There was a possibility to couple separated sections of different birefringent fibers and also to rotate one of the birefringent fibers along its longitudinal axis as it is schematically presented in Fig. 3. Figure 4 presents results of polarization measurements in which an elliptical-core $(4 \times 18 \mu \mathrm{m})$ anisotropic LCF of the length $c a .5 \mathrm{~cm}$ was connected to the HB bow-tie fiber (about $50 \mathrm{~cm}$ long) and both fibers were placed between crossed polarizers whereas their polarization characteristics were investigated. The LCF characterized by homogeneous transverse orientation of LC molecules has a property of single-polarization multimode propagation. The whole manufacturing process of the LCF was described elsewhere [7] and the photograph of the whole PAS-1 system is shown in Fig. 5. 


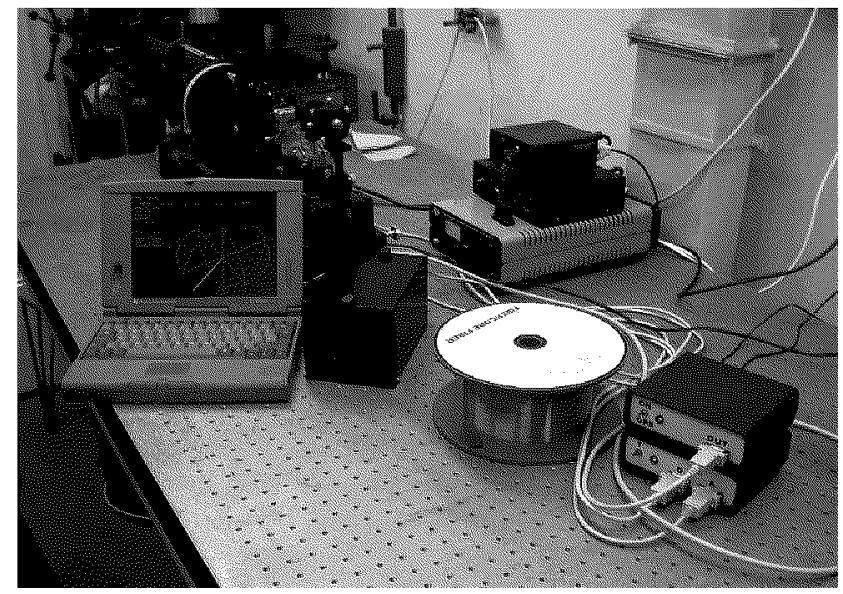

Fig. 5. Measurement apparatus PAS-1 to investigate polarization and depolarization effects in birefringent fibers.

The issue of DOP fading of the light passing through the HB fibers as well as influence of coherence of a light source is of the highest importance in polarimetric fiber optic sensors.

When a HB-600 single-mode bow-tie fiber coupled to the TOLD-9321 Toshiba laser diode $(670 \mathrm{~nm})$ was used, interesting effects have been observed. First, DOP of the light coming out of the fiber significantly decreased with the fiber length. As it can be seen in Figs. 6 and 7 a minimal value of DOP is close to $0.6(0.3 \mathrm{~m}$ long fiber) while it is close to 0.2 for a much longer fiber $(100 \mathrm{~m})$.
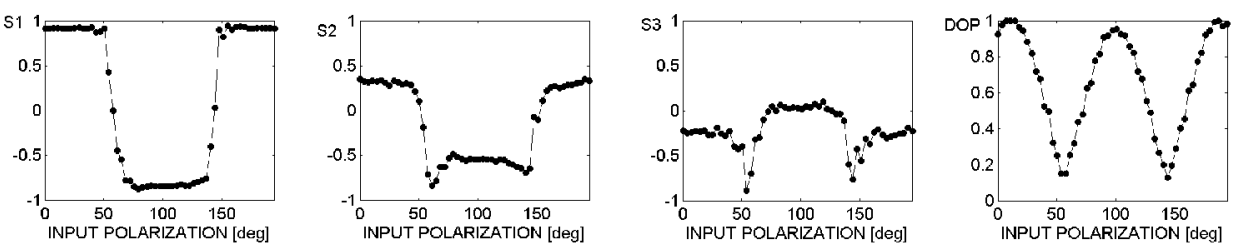

Fig. 6. Stokes parameters and DOP at the output of a $100 \mathrm{~m}$ HB-600 bow-tie fiber coupled to a laser diode (670 nm, TOLD-9321 Toshiba).
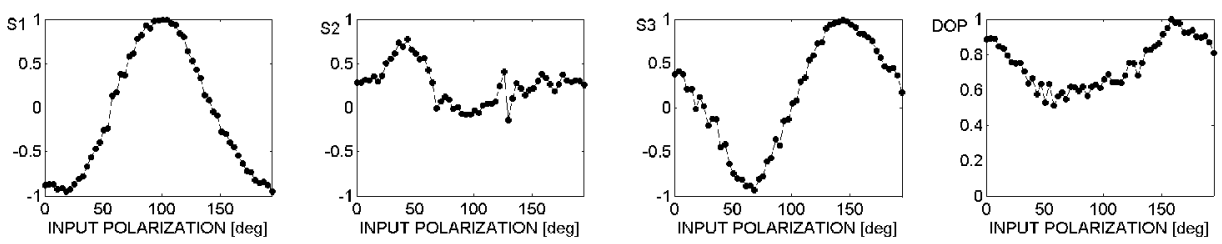

Fig. 7. Stokes parameters and DOP at the output of a $0.3 \mathrm{~m} \mathrm{HB}-600$ bow-tie fiber coupled to a laser diode (670 nm, TOLD-9321 Toshiba). 


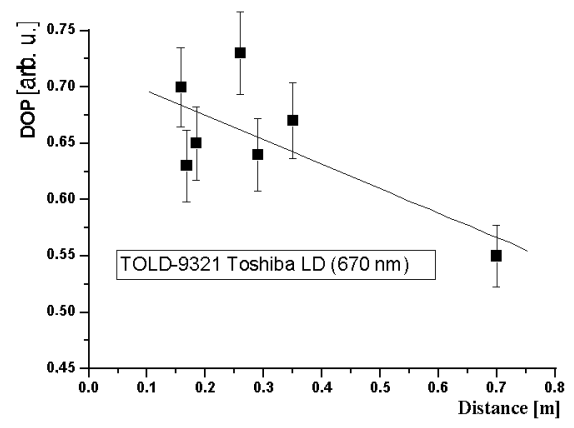

Fig. 8. DOP (input polarization directed at $45^{\circ}$ in respect to birefringence axes) as a function of the fiber length for a HB-600 fiber coupled to TOLD-9321 Toshiba LD.

Figure 8 shows a minimal value of DOP as a function of the fiber length. For a smaller fiber length the DOP of the light has increased. We have roughly estimated a decay of DOP along the fiber introducing a coefficient that defines the slope of linear regression:

$$
\alpha_{\mathrm{DOP}}=\frac{\Delta P_{\mathrm{DOP}}}{\Delta L} \cong-0.15,
$$

where $P_{\text {DOP }}$ is the value of DOP and $L$ is the length of HB fiber.

Otherwise, when a HB-600 single-mode bow-tie fiber was coupled to $\mathrm{He}-\mathrm{Ne}$ laser, the DOP of the light was constant for a measured fiber length. Moreover, long coherence length causes that the propagating light does not loose polarization properties, and the polarimetric sensors have a large signal dynamics. For a short

TABLE

Comparison of polarization properties of single-mode low-birefringence (LB) telecom fibers and polarization-maintaining (PM) HB fibers.

\begin{tabular}{l|c|c}
\hline \hline Beat length & Telecom LB fibers & PM HB fibers \\
\hline $\begin{array}{l}\text { Coherence of the light } \\
\text { source }\end{array}$ & $\begin{array}{c}L_{\mathrm{B}} \sim 10 \mathrm{~m} \text {, random } \\
\text { birefringence axes }\end{array}$ & $\begin{array}{c}L_{\mathrm{B}} \sim 1 \mathrm{~mm} \text {, well-defined } \\
\text { birefringence axes }\end{array}$ \\
\hline First-order PMD & $\underline{\text { not so important }}$ & $\begin{array}{c}\sim 1000 \mathrm{ps} / \mathrm{km} \\
(1 \mathrm{ps} / \mathrm{m})\end{array}$ \\
\hline PMD impact & Limits bit rate & $\begin{array}{c}\text { Limits dynamics } \\
(\text { lowers DOP) }\end{array}$ \\
\hline PMD compensation & $\begin{array}{c}\text { Dynamic (feedback) } \\
\text { control of birefringence \& axes: } \\
\text { HB, LC fibers }\end{array}$ & $\begin{array}{c}\text { (e.g. 90 } \\
\text { splice })\end{array}$ \\
\hline
\end{tabular}


coherence length source (i.e. laser diode), the propagating light looses properties of polarization. This phenomenon introduces a regime to a fiber length for the polarimetric sensors.

Then, the fall of the DOP parameter was particularly significant for angles close to $45^{\circ} \pm 90^{\circ}$ in respect to birefringence axes of the HB fiber. These both effects can be attributed to a limited value of the coherence length that characterize the laser diode used in the measurement and also linked to PMD of the fiber as it was suggested elsewhere [8].

At the end, we present the Table that comparatively summarizes polarization properties of standard single-mode low-birefringence telecom fibers with highly birefringent polarization-maintaining fibers.

\section{Conclusions}

To conclude, we have discussed the phenomenon of polarization mode dispersion in optical fibers characterized by intrinsic birefringence, both linear and nonlinear measured selected polarization parameters of birefringence optical fibers in comparison to standard telecom single-mode fibers. It appeared that birefringence systems composed of elliptical-core LCFs and "solid-core" HB fibers exhibit good polarization-maintaining properties that significantly dependent of the coherence length of the light source used. The long-term aim of these studies is to propose an efficient system with dynamically-controlled birefringence to compensate polarization-mode dispersion in optical telecommunication and in fiber-optic sensing applications.

\section{Acknowledgement}

The work was supported by the State Committee for Scientific Research (KBN) under the grant 8T11D027019.

\section{References}

[1] T.R. Wolinski, Polarimetric Optical Fibers and Sensors, in: Progress in Optics, Ed. E. Wolf, Vol. XL, North Holland, Amsterdam 2000, p. 1.

[2] I.T. Lima, Jr., G. Biondini, B.S. Marks, W.L. Kath, C.R. Menyuk, IEEE Photon. Technol. Lett. 14, 627 (2002).

[3] A.W. Domański, T.R. Woliński, M.A. Karpierz, A. Kujawski, Proc. SPIE 2507, 204 (1995).

[4] M. Born, E. Wolf, Principles of Optics, sixth ed., Pergamon Press, London.

[5] M.N. Islam, C.D. Poole, J.P. Gordon, Opt. Lett. 4, 1011 (1989).

[6] T.R. Woliński, A.W. Domański, P. Lesiak, A. Wyrwas, submitted to IEEE Trans. Instrum. Meas.

[7] A. Szymańska, T.R. Woliński, Mol. Cryst. Liq. Cryst. 375, 723 (2002).

[8] H. Rosenfeldt, R. Urlich, E. Brinkmeyer, U. Feiste, C. Schubert, J. Berger, R. Ludwig, H.G. Weber, A. Ehrhardt, in: Proc. ECOC'01, Amsterdam (Netherlands) 2001, p. 68 . 